\title{
IMPACT OF SPECULAR POINT ESTIMATION INACCURACIES ON TECHODEMOSAT-1 GNSS-REFLECTOMETRY OBSERVABLES OVER OCEANS
}

\author{
A. Grieco G., B. Stoffelen A. \\ Royal Dutch Meteorological Institute \\ R\&D Satellite Observations \\ 3731 GA De Bilt, The Netherlands
}

\begin{abstract}
This paper presents an assessment of the effects of specular point (SP) estimation inaccuracies on the Signal to Noise Ratio peak (SNR ${ }^{P E A K}$ ) used for ocean wind speed retrievals from TechDemoSat-1 (TDS-1) Delay Doppler Maps (DDMs). Results show that the more inaccurate the estimated Doppler frequency at the SP, the lower the intensity of the $\mathrm{SNR}^{P E A K}$. Differences may be up to $2 \mathrm{~dB}$.
\end{abstract}

Index Terms - GNSS-R, SNR peak, Doppler frequency, specular point.

\section{INTRODUCTION}

Global Navigation Satellite System Reflectometry (GNSS-R) from satellite is expected to give reliable wind speed retrievals for assimilation in numerical weather prediction (NWP) models. The required wind speed accuracy for such purposes should be better than $2 \mathrm{~ms}^{-1}$ in the range $0-20 \mathrm{~ms}^{-1}$. To the best of our knowledge, this performance can be obtained only for wind speeds lower than approximately $7 \mathrm{~m} / \mathrm{s}$. It is important, at this stage, that every source of mis-calibration of the DDM observables that are used for wind speed retrievals is properly understood and taken into account.

Recently, it has been demonstrated that the Delay Doppler Maps (DDMs) acquired by the Space GNSS Receiver Remote Sensing Instrument (SGR-ReSI) onboard the polar orbiting satellite TechDemoSat-1 (TDS-1) suffer of distortions that are caused by specular point (SP) estimation inaccuracies [1]. Such inaccuracies are caused by an inaccurate model of the Earth surface, the so called quasi spherical (QS) approximation [2].

The aim of this study is to assess the effects of such distortions on the Signal to Noise Ratio peak ( $\mathrm{SNR}^{P E A K}$ ), which is recognized to be one of the observables used for wind speed retrievals from TDS-1 DDMs [3] [4].

The paper is organized as follows: the dataset used for this study is described in section 2; the methodology is presented in section 3; results are discussed in section 4 and, finally, conclusions and suggestion for future work are reported in section 5 .

\author{
C. Portabella M.
}

\author{
Institute of Marine Sciences (ICM-CSIC) \\ Barcelona Expert Centre (BEC) \\ 08003 Barcelona, Spain
}

\section{DATASET}

The dataset used for this study relates to all the TDS-1 DDMs acquired during the period spanning from July 4, 2015 until June 19, 2016. In this period, the SGR-ReSI was operated in the Fixed Gain Mode (FGM). FGM is more suitable for calibrations purposes since the receiver absolute power levels are known [4].

DDMs have been collocated with all the occurring scatterometer derived winds from Advanced Scatterometer (ASCAT) and RapidScat. ASCAT is operated by the European Agency for the Exploitation of Meteorlogical Satellites (EUMETSAT) onboard the polar orbiting satellites Metop, while RapidScat is operated by the National Aeronautics and Space Administration (NASA) onboard the International Space Agency. The accuracy of such scatterometer derived wind speeds is expected to be within $1 \mathrm{~ms}^{-1}$ [5].

DDMs and scatterometer derived winds have been collocated according to the following spatio-temporal criteria: a) the maximum allowed distance between the TDS-1 SP and the scatterometer wind vector cell (WVC) is within $25 \mathrm{~km}$; b) the maximum temporal lag between TDS-1 and scatterometer acquisitions is within 20 minutes. This criterion has been relaxed to 1.5 hours in order to assess the sensitivity of the $\mathrm{SNR}^{P E A K}$ to SP inaccuracies in such poor temporal collocation requirement.

Finally, all the valid collocations have been added with wind field information from the European Centre for Mid to Short Range Weather Forecasts (ECMWF) 3 hour forecasts. This information has been used for simulating DDMs for a consistency check with TDS-1 DDMs.

The dataset has been quality flagged through the quality control (QC) scheme described in [1]. In particular, all the DDMs that present a poor correlation with the simulated DDMs are filtered out. The correlation threshold has been set up to 0.9. Finally, all DDMs acquired with an incidence angle at the SP higher than $45^{\circ}$ and with a latitude at the SP higher than $80^{\circ} \mathrm{N}$ or lower than $80^{\circ} \mathrm{S}$ have been flagged as poor quality.

The entire good quality flagged dataset consists of about 93,000 couples in the most severe temporal collocation re- 
quirement, which become more than 500,000 when the temporal constrained is relaxed to $1.5 \mathrm{~h}$. The distribution of $\mathrm{SNR}^{P E A K}$ versus wind speed for the entire dataset is represented in Figure 1, where frequencies have been normalized to the maximum value.

\section{METHODOLOGY}

WAVeform simulation in PYthon (WAVPY) [6] is an open source tool that has been designed for analysis and simulation of GNSS-R waveforms (WFs). In this paper, WAVPY has been used to simulate the TDS-1 DDMs using collocated ECMWF wind field information, for a consistency check with real DDMs. Furthermore, it has been used for computing a more accurate SP location through its embedded ellipsoid model of the Earth surface. This SP estimate is considered the reference truth. The absolute value of the difference between the TDS-1 onboard estimation of the Doppler frequency at the SP and the one from WAVPY is computed according to the following formula:

$$
|\Delta f| \approx\left|\frac{\mathbf{V}_{\mathbf{R}} * \mathbf{m}^{\mathbf{T D S}-\mathbf{1}}}{\lambda}-\frac{\mathbf{V}_{\mathbf{R}} * \mathbf{m}^{\mathbf{W P}}}{\lambda}\right|
$$

where $\mathbf{V}_{\mathbf{R}}$ is the receiver velocity vector and $\mathbf{m}^{\mathbf{T D S}-\mathbf{1}}$ $\left(\mathbf{m}^{\mathbf{W P}}\right)$ is the outgoing unit vector from the onboard (WAVPY) estimated SP towards the receiver [1].

$\mathrm{SNR}^{P E A K}$ has been binned in wind speed bins of $1 \mathrm{~ms}^{-1}$ in the range $0-20 \mathrm{~ms}^{-1}$. Outside of this range, the number of samples is too low.

For every bin, the Pierson correlation coefficient and a linear polynomial fit of $\mathrm{SNR}^{P E A K}$ versus $|\triangle f|$ have been computed. For every fit, the p-value associated to the null test has been considered. Finally, a sensitivity analysis with respect to the time collocation requirement has been carried on, by relaxing the temporal constraint to 1.5 hour instead of 20 minutes.

\section{RESULTS}

For the sake of brevity, only one density plot of $\mathrm{SNR}^{P E A K}$ versus $|\Delta f|$ in the wind speed range $6-7 \mathrm{~ms}^{-1}$ is reported for illustrative purposes (Figure 2). It can be seen that the negative trend of $\mathrm{SNR}^{P E A K}$ versus $|\Delta f|$ can be appreciated from a visual check. Here, frequencies have been normalized to the maximum value of the density plot. The linear fitting curve is drawn in dashed black, and its slope coefficient is reported in the legend, together with the p-value associated to the null test and the total number of samples in the bin. It can be seen that the density plot depicted in Figure 2 is in agreement with the general distribution shown in Figure 1.

The distribution of this dataset is constrained by collocations with ASCAT and RapidScat. Indeed, due to orbital reasons, most collocations are located in mid-high latitudes, where the accuracy of the QS approximation implemented onboard for the estimation of the SP is lower, giving rise to errors of the Doppler value at the SP in the order of $200 \mathrm{~Hz}$ [2]. On the contrary, most accurate estimations are located around the equatorial area. It would be interesting doing the same analysis for a more uniform distribution of samples with respect to $|\Delta f|$. In this study, this could be possible by randomly reducing the most populated part of the distribution, but the number of samples would be severely reduced.

In our opinion, a more uniform distribution could be possible for the Cyclone GNSS (CYGNSS) satellite mission [7]. TDS-1 and CYGNSS satellites share a very similar technology, being both manufactured by the Surrey Satellite Technology Limited (SSTL). In particular, the algorithm for the estimation of the SP is identical. Therefore, CYGNSS DDMs are expected to suffer of the same inaccuracies of the SP location. Finally, the huge amount of acquisitions should lead to a much larger number of collocations with scatterometer acquisitions. Such kind of study is left for the next future.

Figure 3 shows a synoptic view of the correlation coefficients for all the wind speed bins. The red curve refers to the optimal temporal collocation requirement with scatterometers (20 min), while the black curve refers to the more relaxed constraint $(1.5 \mathrm{~h})$. It can be seen that, in the wind speed range $0-13 \mathrm{~ms}^{-1}$, the absolute value of the correlation coefficient computed in the more severe temporal constraint is always lower than the one computed in the more relaxed temporal constraint. This plot suggests that a proper temporal collocation constraint is important for investigating some phenomena that, otherwise, will be masked by the uncertainty introduced by a poorer collocation.

For all the bins in the range $0-13 \mathrm{~ms}^{-1}$, the absolute value of the slope coefficient is greater or equal to $5^{*} 10^{-3} \mathrm{~dB} \mathrm{~Hz}^{-1}$ (even if $\mathrm{Hz}^{-1}$ is equivalent to $\mathrm{s}$, here we want to emphasizes the dependency of SNR ${ }^{P E A K}$ on the Doppler frequency shift). This value is equivalent to $1 \mathrm{~dB}$ for a Doppler shift of $200 \mathrm{~Hz}$. It's important to stress, once more, that such Doppler shift is highly likelihood at mid-high latitudes.

In order to further remark the presence of such inaccuracies, Figure 4 shows the average $\mathrm{SNR}^{P E A K}$ versus wind speed, when $\mathrm{SNR}^{P E A K}$ is binned also for Doppler shift of $30 \mathrm{~Hz}$, in the range $0-210 \mathrm{~Hz}$. It can be seen that the curves clearly spread. In particular, one can appreciate that the orange curve (bin 30-60 Hz) and the light violet curve (bin 180-210 Hz) never cross each other, the first one being always higher than the second one. Therefore, the higher the errors in Doppler frequency at SP, the lower the $\mathrm{SNR}^{P E A K}$. Furthermore, differences may be up to $2 \mathrm{~dB}$. Finally, the black dashed line represents the average $\mathrm{SNR}^{P E A K}$ versus wind speed for the entire dataset. It can be seen that this curve is in the lowest part of the plot, very close to the curves relating to bins with highest Doppler shift. Once more, this is consistent with the distribution of the dataset shown in Figure 1. 


\section{CONCLUSIONS AND FUTURE WORK}

An analysis on how specular point (SP) inaccuracies affect delay-Doppler map (DDM) observable Signal to Noise Ratio peak $\left(\mathrm{SNR}^{P E A K}\right)$ of the satellite mission TechdemoSat 1 (TDS-1) has been presented.

Results show that such inaccuracies lead to errors in the $\mathrm{SNR}^{P E A K}$ that may be larger than $1 \mathrm{~dB}$. It is recommended that such inaccuracies are properly taken into account because they may heavily impact wind speed retrievals. This is particularly noticeable for wind speed higher than $7 \mathrm{~ms}^{-1}$, due to the lower sensitivity of SNR ${ }^{P E A K}$ to wind speed. The way in which this should be done is beyond the scope of this paper, and is left for future studies.

The dataset presented in this paper is highly conditioned by TDS- 1 collocations with scatterometer acquisitions from ASCAT and RapidScat. These collocations are more frequent in mid-high latitudes, where SP inaccuracies are larger. For this purpose, in the next future, this analysis will be also carried on for Cyclone GNSS (CYGNSS) acquisitions. Indeed, CYGNSS level 1 processor uses the same quasi-spherical (QS) approximation used in TDS-1 mission for the estimation of the SP location, therefore, they are suspected of the same kind of distortions as TDS-1. Furthermore, due to the huge number of acquisitions from CYGNSS satellites, the number of collocations with scatterometer acquisitions is expected to be much larger than for TDS- 1 and, therefore, it will be possible to extract a more suitable dataset.

Finally, this study suggests that future GNSS-R missions will consider to operationally deliver raw data in order to easily reprocess previous acquisitions when a source of inaccuracies is detected.

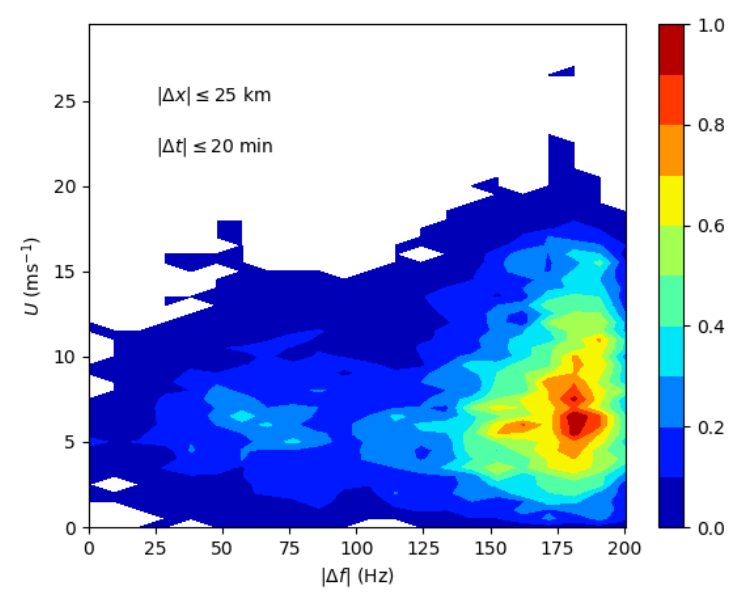

Fig. 1. Distribution of scatterometer wind speed (y-axis) versus absolute value of the difference between the estimated and the true Doppler frequencies at the specular point (x-axis). Frequencies are normalized.

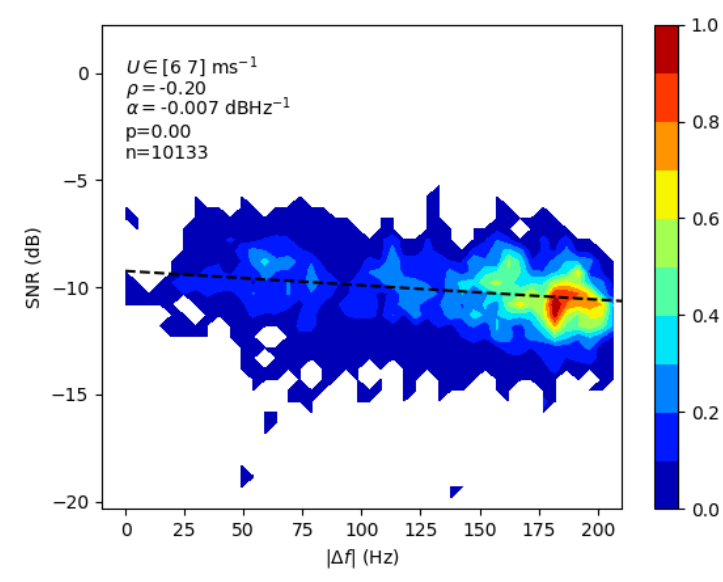

Fig. 2. Density plot of $\mathrm{SNR}^{P E A K}$ (y-axis) versus $|\Delta f|(\mathrm{x}-$ axis) in the wind speed range $6-7 \mathrm{~ms}^{-1}$. Frequencies are normalized.

\section{REFERENCES}

[1] G. Grieco, A. Stoffelen, M. Portabella, M. B. Rivas, W. Lin, and F. Fabra, "Quality control of delay-doppler maps for stare processing," IEEE Transactions on Geoscience and Remote Sensing, pp. 1-11, 2018.

[2] P. Jales, Spaceborne receiver design for scatterometric GNSS reflectometry, Ph.D. thesis, Univ. of Surrey, 2012.

[3] S. Soisuvarn, Z. Jelenak, F. Said, P. S. Chang, and A. Egido, "The gnss reflectometry response to the ocean surface winds and waves," IEEE Journal of Selected Topics in Applied Earth Observations and Remote Sensing, vol. 9, no. 10, pp. 4678-4699, Oct 2016.

[4] W. Lin, M. Portabella, G. Foti, A. Stoffelen, C. Gommenginger, and Y. He, "Toward the generation of a wind geophysical model function for spaceborne gnss-r," IEEE Transactions on Geoscience and Remote Sensing, pp. 112, 2018.

[5] A. Stoffelen, J. A. Verspeek, J. Vogelzang, and A. Verhoef, "The cmod7 geophysical model function for ascat and ers wind retrievals," IEEE Journal of Selected Topics in Applied Earth Observations and Remote Sensing, vol. 10, no. 5, pp. 2123-2134, May 2017.

[6] F. Fabra, E. Cardellach, W. Li, and A. Rius, "Wavpy: A gnss-r open source software library for data analysis and simulation," in 2017 IEEE International Geoscience and Remote Sensing Symposium (IGARSS), July 2017, pp. 4125-4128.

[7] Christopher S. Ruf, Robert Atlas, Paul S. Chang, Maria Paola Clarizia, James L. Garrison, Scott Gleason, 


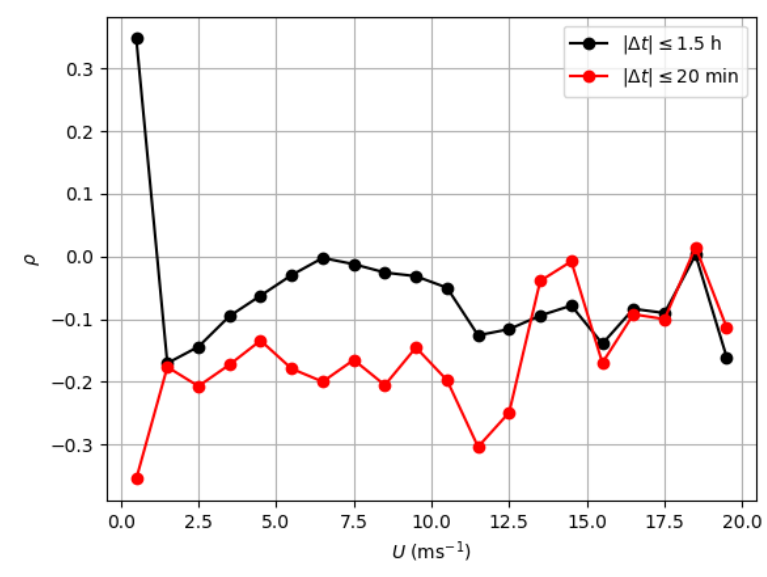

Fig. 3. Pierson correlation coefficient between $\mathrm{SNR}^{P E A K}$ and $|\Delta f|$ for every $1 \mathrm{~ms}^{-1}$ wind speed bin in the range $0-20$ $\mathrm{ms}^{-1}$. Black curve: temporal distance between scatterometer and TDS-1 acquisition within $1.5 \mathrm{~h}$. Red curve: temporal distance within $20 \mathrm{~min}$.

Stephen J. Katzberg, Zorana Jelenak, Joel T. Johnson, Sharanya J. Majumdar, Andrew Obrien, Derek J. Posselt, Aaron J. Ridley, Randall J. Rose, and Valery U. Zavorotny, "New ocean winds satellite mission to probe hurricanes and tropical convection," Bulletin of the American Meteorological Society, vol. 97, no. 3, pp. 385-395, 2016.

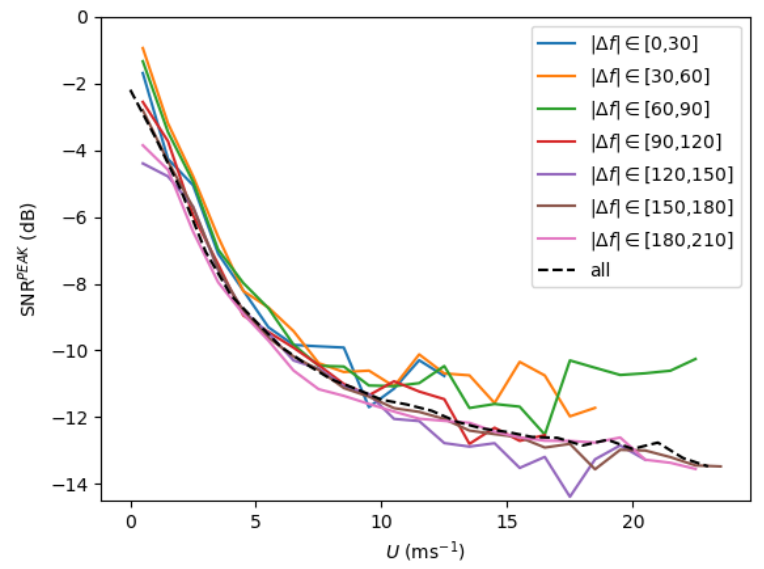

Fig. 4. Average $\mathrm{SNR}^{P E A K}$ versus wind speed separated according to $|\Delta f|$. Black dotted curve: average $\mathrm{SNR}^{P E A K}$ versus wind speed for the entire dataset 